Research Paper

\title{
Evaluation of 7th Edition of AJCC Staging System for Nasopharyngeal Carcinoma
}

\author{
YuFeng Ren ${ }^{*}$, Huizhi Qiu ${ }^{2 *}$, Yujie Yuan³, Jinning Ye3, Yunhong Tian², Bixiu Wen', Weijun Zhang ${ }^{2}$ and Qun \\ $\mathrm{Li}^{4}, 5 \bowtie$ \\ 1. Department of Radiation Oncology, The First Affiliated Hospital, Sun Yat-sen University, Guangzhou 510080, P.R.China; \\ 2. Department of Radiotherapy, cancer center of Guangzhou medical university, Guangzhou 510080, P.R.China; \\ 3. Center of Gastrointestinal Surgery, the First Affiliated Hospital, Sun Yat-sen University, Guangzhou 510080, P.R.China; \\ 4. Department of Radiation Oncology, Sun Yat-sen University Cancer Center, Guangzhou 510060, P.R.China; \\ 5. Sun Yat-sen University Cancer Center, State Key Laboratory of Oncology in South China, Collaborative Innovation Center for Cancer Medicine, Guangzhou \\ 510060, Guangdong, P. R. China. \\ * These authors contributed equally to this work.
}

$\triangle$ Corresponding author: Qun Li., M.D., Ph.D; Sun Yat-sen University Cancer Center, State Key Laboratory of Oncology in South China, Collaborative Innovation Center for Cancer Medicine, Guangzhou 510060, Guangdong, P. R. China; Department of Radiation Oncology, Cancer Center, Sun Yat-sen University, Guangzhou 510060, P.R.China. E-mail: liqun@sysucc.org.cn

(C) Ivyspring International Publisher. This is an open access article distributed under the terms of the Creative Commons Attribution (CC BY-NC) license (https://creativecommons.org/licenses/by-nc/4.0/). See http://ivyspring.com/terms for full terms and conditions.

Received: 2017.01.15; Accepted: 2017.03.31; Published: 2017.06.03

\begin{abstract}
Purpose: To evaluate and improve the $7^{\text {th }}$ edition International Union against Cancer/American Joint Committee on Cancer staging system for nasopharyngeal carcinoma.

Methods: A retrospective review of the data from 905 patients with biopsy-proven non-disseminated nasopharyngeal carcinoma was performed. All the patients were examined by magnetic resonance imaging (MRI) and received radiotherapy.

Results: Satisfied distributions among the stages were observed in the $7^{\text {th }}$ edition staging systems. LRFS only differed in classifications betweenTl and $T 3, T 1$ and $T 4 \quad(P=0.022$ and $P=0.016$, respectively). Significant differences were observed between patients without and with masticator space involvement for OS, DMFS and PFS $(p<0.05)$. No statistically significant differences in LRFS were observed among different groups with anatomical masticator space involvement. The DMFS between $\mathrm{N} 2$ and $\mathrm{N} 3 \mathrm{~b}, \mathrm{~N} 3 \mathrm{a}$ and $\mathrm{N} 3 \mathrm{~b}$ were lack of significance $(\mathrm{P}=0.060$ and $\mathrm{P}=0.59)$. The $T$ category and $\mathrm{N}$ category were independent prognostic factors for the major endpoints in the Cox multivariate regression analysis $(\mathrm{P}<0.01)$.

Conclusion: This study confirmed the prognostic value of the $7^{\text {th }}$ edition UICC/AJCC staging system, the revisions of the $7^{\text {th }}$ edition staging system are acceptable. However, our study also revealed limitations in the current staging system and suggested some potential modifications in future revision.
\end{abstract}

Key words: Nasopharyngeal carcinoma, American Joint Committee on Cancer, Staging system, prognosis.

\section{Introduction}

Nasopharyngeal carcinoma (NPC) is one of the most prevalent malignancies of head and neck in Southeast Asia and southern China, with a high incidence of about $25-50 / 10^{5}$ individuals per year [1-2]. Distant metastasis and recurrence occur frequently in NPC after treatment [3-5]. The nasopharyngeal carcinoma TNM staging system is crucial for evaluating the prognosis, guiding treatment strategy for different risk groups and facilitating exchanging experiences between multiple treatment centers [6]. However, there was no worldwide accepted staging system for NPC until the merge of the International Union against Cancer (UICC) and the American Joint Committee on Cancer (AJCC). UICC/AJCC staging 
system, amalgamating both Western and Eastern experiences and advocating the staging system, was widely used in the past several decades. The stage assignment and disease prognosis are affected by the improvement of diagnostic and therapeutic technology [7]. Revision is a factor that makes the TNM system the most clinically useful staging system and accounts for its worldwide use ${ }^{[2]}$. Thus, the UICC and AJCC periodically modify the TNM system in response to newly acquired clinical data and prognostic factors ${ }^{[6]}$.

The most recently advocated $7^{\text {th }}$ edition of UICC/AJCC for nasopharyngeal carcinoma is a common set of recommendations from the revision of the previous $6^{\text {th }}$ edition. Several recommendations that derived from the $6^{\text {th }}$ edition [8-9] have been advocated. Patients with oropharynx and nasal fossa involvement, which were T2a category in the $6^{\text {th }}$ edition, are reclassified to T1. Anatomic masticator space involvement is categorized into T4. In the $6^{\text {th }}$ edition UICC/AJCC staging system, the term "masticator space", defined as extension beyond the anterior surface of the lateral pterygoid muscle or beyond the posterolateral wall of the maxillary antrum and/or the pterygomaxillary fissure, was a synonym of infratemporal fossa. Compared with the $6^{\text {th }}$ edition, the $7^{\text {th }}$ edition adopted the description "primarily the muscles of mastication (the medial and lateral pterygoid, masseter and temporalis) enclosed by the superficial layer of the deep cervical fascia", as defined in classical radiological textbooks. Retropharyngeal lymph node is also clarified in the $7^{\text {th }}$ edition staging system. Retropharyngeal lymph node invasion, regardless of unilateral or bilateral location, is staged as N1.

Several studies have confirmed that the current $7^{\text {th }}$ edition staging system for NPC is acceptable, though there are limitations that need further modification [10-11]. However, the follow-up periods of these studies were relatively short. In this retrospective study, we aimed to assess the prognostic value of the $7^{\text {th }}$ edition staging systems in nasopharyngeal carcinoma with a long-term follow-up.

\section{Materials and Methods}

\section{Patients' characteristics}

Between October 2004 and December 2005, 905 patients with newly diagnosed, untreated, non-disseminated NPC were included in this study. All patients underwent a pretreatment evaluation that includes a complete patient history, physical and neurological examinations, hematologic and biochemistry profiles, and MRI of the nasopharynx and neck, chest radiography and abdominal ultrasonography. Medical records and imaging studies were analyzed retrospectively, and the NPC stages of all patients were classified according to the $7^{\text {th }}$ edition of the UICC/AJCC staging system.

\section{Treatment}

Among these 905 patients, 520 (57.5\%) patients underwent conventional two-dimensional radiation therapy, $246 \quad(27.2 \%)$ patients underwent three-dimensional conformal radiation therapy (3D-CRT), and 139 (15.4\%) patients underwent intensity-modulated radiation therapy (IMRT). The techniques of thermoplastic mask, low melting-point lead block, multi-leaf collimator (MLC) and source axis distance (SAD) were applied to radiotherapy. 6-8 MV supervoltage $X$ rays generated by a linear accelerator were used for external irradiation. During the two-dimensional conformal radiotherapy, conventional fraction was given, and the dose for the primary lesion in the nasopharynx was $66 \sim 76$ Gy/33 $\sim 38 \mathrm{~F}$. The dose for the cervical lymphatic drainage area was $50 \sim 66 \mathrm{~Gy} / 25 \sim 33 \mathrm{~F}$. supervoltage $\mathrm{X}$ rays added beta-rays were used to compensate the dose in consideration of skin and subcutaneous tissues in the neck. The prescribed radiation dosage of 3D-CRT were defined as follows: GTVnx (nasopharynx gross tumor volume): 65-70 Gy; GTVnd (positive neck lymph nodes volume): 60-70 Gy; CTV60 (clinical target volume 60): 60 Gy; CTVnx50 (nasopharynx clinical target volume 50): 50 Gy; CTVnd50 (neck nodal clinical target volume): $50 \mathrm{~Gy}$. The prescribed radiation dosage of IMRT was defined as follows: 68 Gy in 30 fractions to the planning target volume (PTV) of the primary gross tumor volume (GTV-P), 60 to 64 Gy to the PTV of nodal gross tumor volume (GTV-N), 60 Gy to the PTV of CTV-1, and 54 Gy to the PTV of CTV-2 and CTV-N. In case of residual tumor confined in the nasopharyngeal cavity after external irradiation, afterloading irradiation was given at the dosage of 10-15Gy by 2-3 fraction. A total of 655 $(72.4 \%)$ patients received chemotherapy, including various regimens of concurrent chemotherapy in combination with either neoadjuvant chemotherapy or adjuvant chemotherapy. Neoadjuvant or adjuvant chemotherapy consisted of two cycles of cisplatin with 5-fluorouracil or cisplatin with taxanes for every 3 weeks. Concomitant chemotherapy consisted of cisplatin given on weeks 1,4 , and 7 during the radiotherapy (RT) process, or cisplatin given weekly (Table 1). Salvage treatments (including intracavitary brachytherapy, surgery, and chemotherapy) were provided in the events of documented relapse or persistent disease when necessary. Details of the radiation therapeutic techniques used at the Cancer 
Center of Sun Yat-sen University have been previously described ${ }^{[12-14]}$.

Table 1. Characteristics of 905 patients

\begin{tabular}{ll}
\hline & $\mathrm{N}=905$ \\
\hline Sex & \\
male & $698(77.2 \%)$ \\
Agemale & $207(22.8 \%)$ \\
Median age & \\
range & 46 \\
ECOG PS & $16-78$ \\
$0-1$ & \\
2 & $886(97.9 \%)$ \\
Histological type & $19(2.1 \%)$ \\
Who I & \\
Who II/III & $38(4.2 \%)$ \\
Radiotherapy & $867(95.8 \%)$ \\
Two-dimension radiotherapy & \\
Three- dimension radiotherapy & $520(57.5 \%)$ \\
IMRT & $246(27.2 \%)$ \\
RT dose (Gy) & $13915.3 \%)$ \\
nasopharynx & \\
lymphonode & $70(66-76)$ \\
Chemotherapy & $60(50-66)$ \\
no & \\
induction & $250(27.7 \%)$ \\
induction +CCRT & $280(30.9 \%)$ \\
induction +CCRT +adjuvant & $128(14.2 \%)$ \\
induction + adjuvant & $19(2.1 \%)$ \\
CCRT & $12(1.3 \%)$ \\
CCRT +adjuvant & $191(21.2 \%)$ \\
adjuvant & $21(2.3 \%)$ \\
\hline & $3(0.3 \%)$ \\
\hline
\end{tabular}

\section{Follow-up and Statistical analysis}

The follow-up duration was calculated from the first day of treatment to either the day of death or the day of the last follow-up. Patients were examined at least every 3 months during the first 2 years; thereafter, a follow-up examination was performed every 6 months for up to 5 years or until death. The time of last follow-up was January, 2015 and the median follow-up period was 86.4 months (range, 1.4-120.4 months).

All events were measured from the date of commencement of treatment. The following end points (time to the first defining event) were assessed: OS, LRFS, DMFS, and PFS. Local recurrence was confirmed by fiberoptic endoscopy and biopsy or only MRI. Distant metastases were diagnosed on the basis of clinical symptoms, physical examination, and imaging, including chest radiography, bone scanning, MRI, and abdominal sonography.

All statistical analyses were conducted with Statistical Package for the Social Sciences (SPSS) 16.0 software (SPSS Inc., Chicago, IL). The survival rates were calculated by the Kaplan-Meier method, and survival differences were compared by the log-rank test. Multivariate analyses with the Cox proportional hazards model were used to calculate the hazard ratio (HR) and test independent significance by backward elimination of insignificant explanatory variables. Host factors (age and sex) were included as covariates in all tests. The criterion for statistical significance was set at $\mathrm{p}<0.05,2$-tailed T-test.

\section{Results}

\section{Patient characteristics and treatment toxicity}

According to the $7^{\text {th }}$ edition of UICC/AJCC staging system, balanced distributions could be achieved among different stages. The distribution of the stage groups for the whole series was $6.0 \%$ for stage I, $22.1 \%$ for stage II, $35.7 \%$ for stage III, $30.9 \%$ for stage IVa, and $5.3 \%$ for stage IVb. That is, $28.1 \%$ of patients were classified as early-stage (stage I and stage II), and $71.9 \%$ of patients were classified as advanced-stage (stage III and IV). Table 2 lists the T and $\mathrm{N}$ distribution data. Among the 905 patients, the 10 -year OS, LRFS, DMFS and PFS were $68 \%, 87 \%, 85 \%$ and $75 \%$, respectively. Altogether 256 patients died before the end of the study, 91 patients experienced recurrence, 125 patients experienced distant metastases, and 12 patients experienced both locoregional recurrence and distant metastases. Late toxicities of cervical subcutaneous fibrosis, xerostomia, trismus, otologic toxicities and radiation encephalopathy could be evaluated in 832, 507, 72, 660 and 63 patients, respectively.

Table 2. Distribution of $T$ categories and $N$ categories of the $7^{\text {th }}$ edition UICC/AJCC NPC staging system

\begin{tabular}{lllllll}
\hline \multicolumn{7}{c}{ UICC2009T } \\
\cline { 2 - 6 } & & 1 & 2 & 3 & 4 & Total \\
\hline UICC2009 N & 0 & $54(6)$ & $30(3.3)$ & $47(5.2)$ & $50(5.5)$ & $181(20)$ \\
& 1 & $75(8.3)$ & $96(10.6)$ & $141(15.6)$ & $165(18.2)$ & $477(52.7)$ \\
& 2 & $28(3.1)$ & $41(4.5)$ & $65(7.2)$ & $65(7.2)$ & $199(22)$ \\
& $3 a$ & $1(0.1)$ & $3(0.3)$ & $9(1.0)$ & $5(0.6)$ & $18(2)$ \\
Total & $3 b$ & $7(0.8)$ & $8(0.9)$ & $10(1.1)$ & $5(0.6)$ & $30(3.3)$ \\
\hline
\end{tabular}

\section{Prognostic value of $\mathbf{T}$ classification}

According to the $7^{\text {th }}$ edition staging system, there were significant differences of OS between classifications $\mathrm{T} 1$ and $\mathrm{T} 3$, classifications $\mathrm{T} 1$ and $\mathrm{T} 4$, classifications $\mathrm{T} 2$ and $\mathrm{T} 3$, and classifications $\mathrm{T} 2$ and $\mathrm{T} 4$ $(\mathrm{P}<0.001$ for all models, details shown in figure 1a). However, LRFS only differed between classifications $\mathrm{T} 1$ and $\mathrm{T} 3, \mathrm{~T} 1$ and $\mathrm{T} 4 \quad(\mathrm{P}=0.022$ and $\mathrm{P}=0.016$, respectively). A marginal difference of LRFS was observed between classifications T2 and T3, T2 and T4 $(\mathrm{P}=0.051$ and $\mathrm{P}=0.047$, respectively, details shown in figure $1 b)$. 

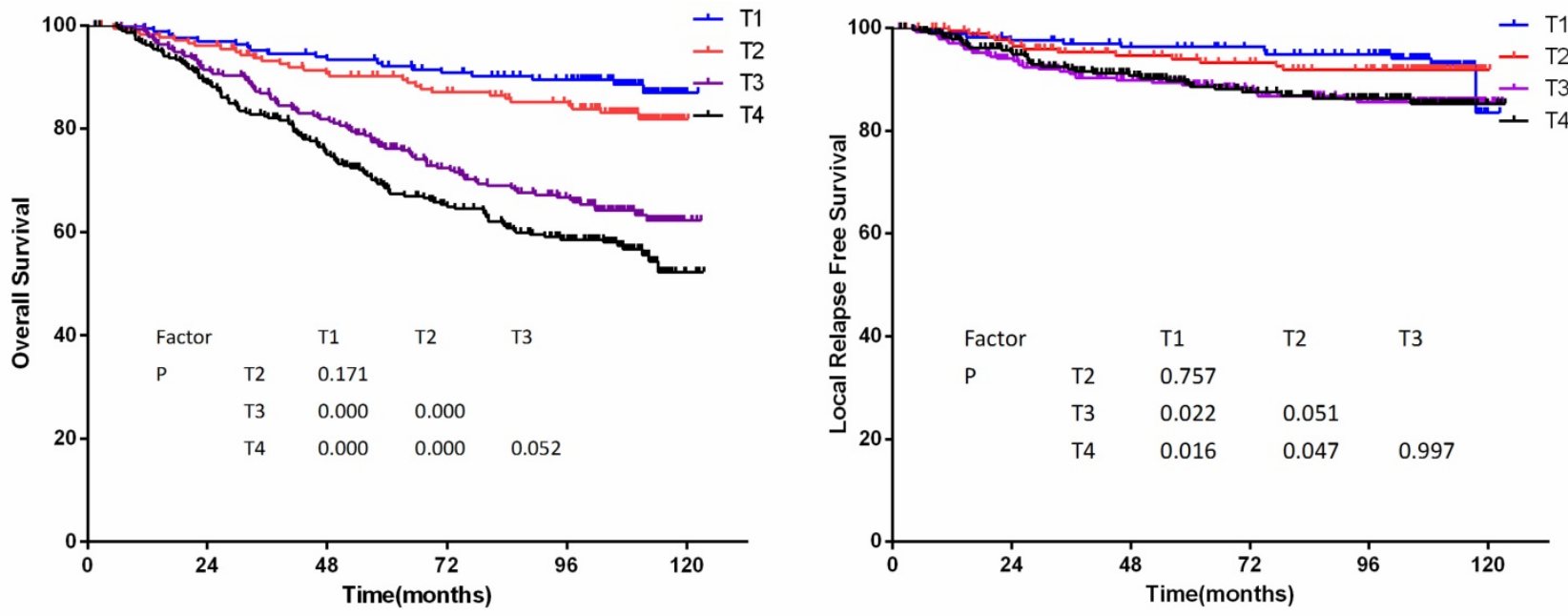

Figure 1. (a) Overall survival and (b)Local relapse-free survival by T-category in 905 non-disseminated patients

The $\mathrm{T}$ classification was an independent prognostic factor of local treatment failure and overall treatment failure in COX multivariate regression analysis $(\mathrm{P}<0.01)$. Hazard ratio of $\mathrm{T}$ categories by multivariate analysis was showed in Table 3.

Table 3. Hazard ratio of $\mathrm{T}$ categories by multivariate analysis

\begin{tabular}{lll}
\hline T category & $\begin{array}{l}\text { Disease failure } \\
\text { HR }(95 \% \mathrm{CI})\end{array}$ & Local failure \\
\hline $\mathrm{T} 1$ & 1 & 1 \\
$\mathrm{~T} 2$ & $1.266(0.707-2.268)$ & $1.151(0.516-2.569)$ \\
$\mathrm{T} 3$ & $2.711(1.657-4.435)$ & $2.185(1.107-4.315)$ \\
$\mathrm{T} 4$ & $2.847(1.744-4.464)$ & $2.205(1.116-4.357)$ \\
\hline
\end{tabular}

Significant differences were observed between patients without and with masticator space involvement for overall survival (72\% vs. $52 \%$, $\mathrm{p}<0.01)$, distant metastasis-free survival $(87 \%$ vs. $81 \%$, $\mathrm{p}=0.032)$ and progress-free survival $(77 \%$ vs. $70 \%$, $\mathrm{p}=0.018$ ). There were no differences observed for local relapse-free survival $(88 \%$ vs. $86 \%$, respectively; $\mathrm{p}=0.474$ ). In addition, in order to investigate the prognostic significance and various classifications for anatomic masticator space involvement (MSI), patients with anatomic masticator space invasion were divided into two groups: Group 1, with medial and/or lateral pterygoid muscle involvement; Group 2 , with infratemporal fossa involvement. There were no statistically significant differences between those groups in LRFS, DMFS and PFS $(\mathrm{P}=0.234, \mathrm{P}=0.075$ and $\mathrm{P}=0.062$, respectively), except OS $(\mathrm{p}=0.002)$. To verify the effect of different MSI classification on patients, patients treated with 3D-CRT/IMRT and patients treated with 2D-CRT were analyzed separately. There were 385 cases in 3D-CRT/IMRT population, 73 with medial and/or lateral pterygoid muscle involvement and 14 with infratemporal fossa involvement. The result showed no statistical significances among LRFS $(p=0.904)$, DMFS $(p=0.158)$ and PFS $(p=0.233)$, while a marginal significant difference in OS $(p=0.046)$ was observed. However, in the 2D-CRT group, significant differences in LRFS $(p=0.036)$ and OS $(p=0.015)$ were observed.

\section{Prognostic value of $\mathbf{N}$ classification}

The long-term DMFS and PFS show well segregation between $\mathrm{N}$ classifications. The N3a has a lower OS and DMFS than N3b. However, the reliability of these results might be limited because of the small number of patients within these subgroups.

According to the $7^{\text {th }}$ edition staging system, significant differences of OS were achieved between $\mathrm{N}$ subsets except for those of classifications $\mathrm{N} 1$ and $\mathrm{N} 2$, classifications $\mathrm{N} 3 \mathrm{a}$ and $\mathrm{N} 3 \mathrm{~b}(\mathrm{P}=0.069$ and $\mathrm{P}$ $=0.903$, details shown in figure $2 a$ ), and significant differences of DMFS were achieved except for those of classifications $\mathrm{N} 2$ and N3b, classifications N3a and $\mathrm{N} 3 \mathrm{~b}(\mathrm{P}=0.060$ and $\mathrm{P}=0.590$, details were shown in figure $2 \boldsymbol{b}$ ). Hazard ratio of $\mathrm{N}$ categories by multivariate analysis was showed in Table 4.

The $\mathrm{N}$ classification was an independent prognostic factor for distant and overall failure in COX multivariate regression $(\mathrm{P}<0.001)$.

Table 4. Hazard ratio of $\mathrm{N}$ categories by multivariate analysis

\begin{tabular}{lll}
\hline Ncategory & $\begin{array}{l}\text { Disease } \\
\text { HR }(95 \% \mathrm{CI})\end{array}$ & Distant failure \\
\hline N0 & 1 & 1 \\
N1 & $2.030(1.282-3.215)$ & $3.647(1.668-7.973)$ \\
N2 & $2.792(1.704-4.572)$ & $5.980(2.674-13.373)$ \\
N3a & $6.249(2.873-13.592)$ & $16.203(5.866-44.756)$ \\
N3b & $4.886(2.416-9.883)$ & $11.943(4.542-31.405)$ \\
\hline
\end{tabular}



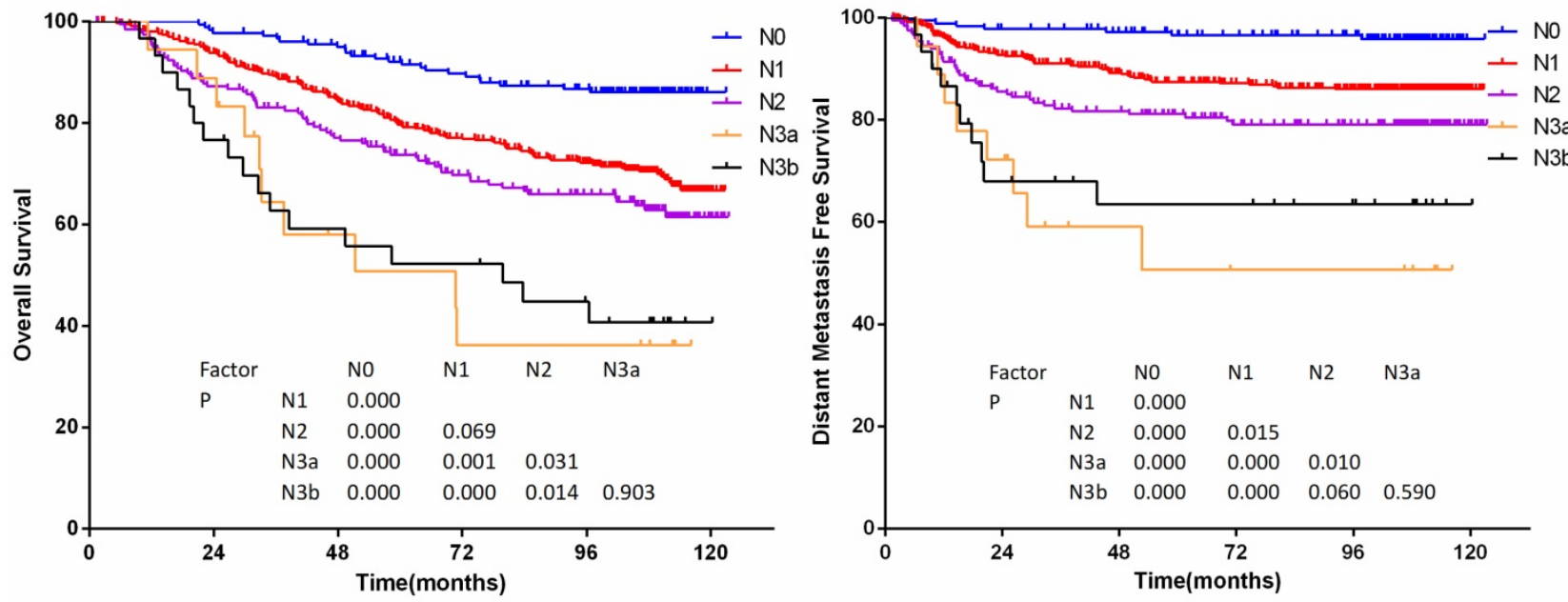

Figure 2. (a)Overall survival and (b)distant metastasis-free survival by $\mathrm{N}$-category in 905 non-disseminated patients
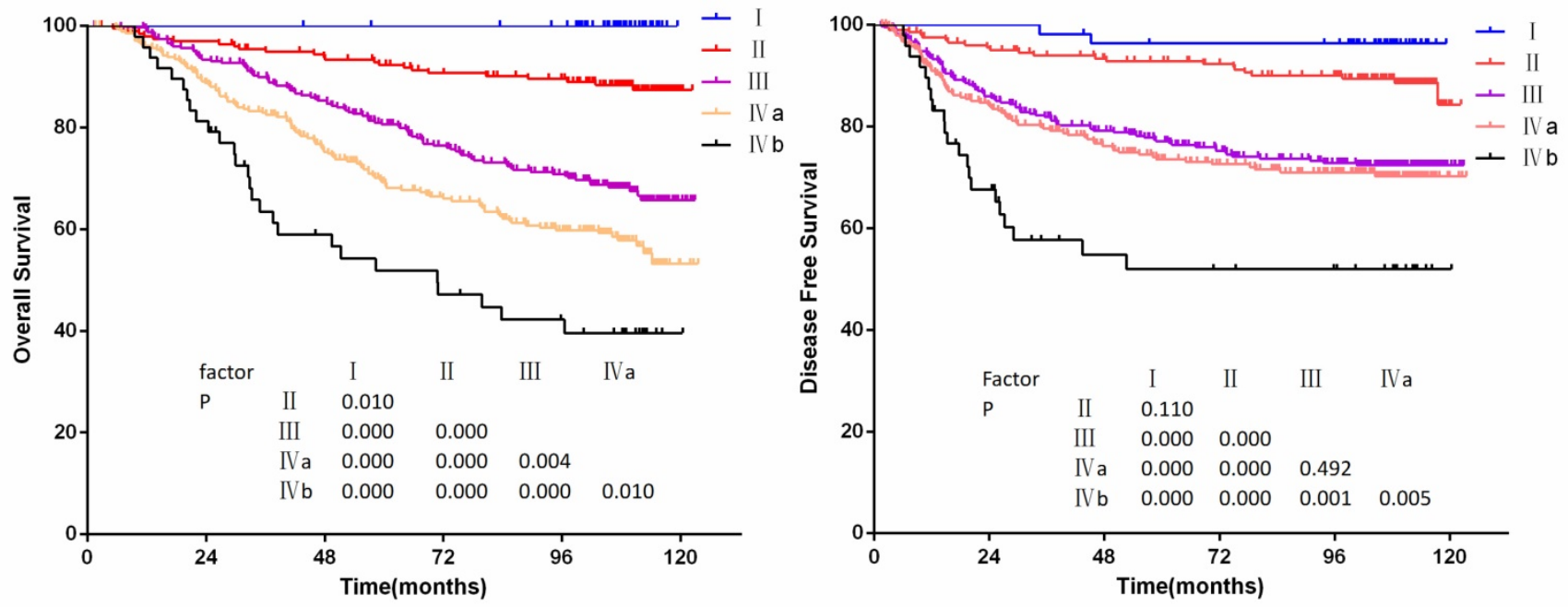

Figure 3. (a) Overall survival and (b)disease-free survival by stage groups in 905 non-disseminated patients

\section{Prognostic value of stage grouping}

The OS and PFS curves for clinical stage are shown in figure $3 a$ to $3 b$. No deaths occurred in stage I patients. The 10-year overall survival rates of stage I-IVb patients were $100 \%, 87 \%, 65 \%, 54 \%, 40 \%$, respectively. Significant differences of OS for clinical stage were achieved $(\mathrm{P}<0.05$ for all models), but there were no significant differences of PFS between stages I and II, stages III and IVa ( $\mathrm{P}=0.110$ and $\mathrm{P}=0.492)$.

\section{Multivariate analysis}

The Cox proportion hazard model for multivariate analysis: age ( $\leq 46$ years vs. $>46$ years), gender (female vs. male), $\mathrm{T}$ categories, $\mathrm{N}$ categories, chemotherapy (no vs. yes), and radiation techniques (2D-CRT vs. 3D-CRT/IMRT). The results showed that $\mathrm{T}$ categories were independent prognostic factors for OS, LRFS, DMFS and PFS in the Cox multivariate regression analysis $(\mathrm{P}<0.005)$. Moreover, $\mathrm{N}$ categories were independent prognostic factors for OS, DMFS and PFS. However, $\mathrm{N}$ categories were not independent prognostic factors for LRFS. The results of the multivariate analysis were shown in Table 5.

Table 5. Summary of multivariate analysis of prognostic factors for the $7^{\text {th }}$ edition UICC/AJCC NPC staging system

\begin{tabular}{llllll}
\hline Endpoint & Factor & $\mathrm{B}$ & $\mathrm{P}$ & $\operatorname{Exp}(\mathrm{B})$ & $\operatorname{Exp}(\mathrm{B}) 95 \% \mathrm{CI}$ \\
\hline OS & age & -0.367 & 0.023 & 0.693 & $0.504-0.952$ \\
& sex & 0.032 & 0.000 & 1.033 & $1.022-1.044$ \\
& T category & 0.518 & 0.000 & 1.679 & $1.472-1.916$ \\
\multirow{3}{*}{ LRFS } & N category & 0.461 & 0.000 & 1.586 & $1.405-1.790$ \\
& sex & -0.502 & 0.075 & 0.605 & $0.348-1.053$ \\
\multirow{2}{*}{ DMFS } & T category & 0.281 & 0.006 & 1.325 & $1.085-1.616$ \\
& Age & 0.019 & 0.015 & 1.019 & $1.004-1.034$ \\
& T category & 0.370 & 0.000 & 1.448 & $1.209-1.735$ \\
DFS & N category & 0.571 & 0.000 & 1.769 & $1.507-2.077$ \\
& sex & -0.305 & 0.088 & 0.737 & $0.519-1.046$ \\
& age & 0.014 & 0.019 & 1.014 & $1.002-1.027$ \\
& T category & 0.358 & 0.000 & 1.431 & $1.244-1.645$ \\
& N category & 0.417 & 0.000 & 1.517 & $1.322-1.741$ \\
\hline
\end{tabular}




\section{Discussion}

A reasonable staging system should offer balanced distributions, obvious hazard discriminations, reasonable hazard predictions and good outcome predictions. Previous retrospective studies have showed that the current $7^{\text {th }}$ edition of the UICC/AJCC staging system for NPC is useful in prognosis, and its superiority over the $6^{\text {th }}$ edition staging system has been reported[10-11]. Our data confirmed that it is an acceptable system for most of our studies. However, some unsatisfactory aspects were revealed, especially due to lack of separation for the major endpoints among the $\mathrm{T}$ categories and lack of hazardous discrimination for DMFS between N3a and N3b.

For $\mathbf{T}$ categories, our data has showed that patients with oropharynx/nasal cavity erosion had better prognosis than patients with parapharyngeal space involvement. Previous studies reported lack of significant differences in LRFS among $\mathrm{T}$ stages according to the $6^{\text {th }}$ edition system [15-16]. When using the $6^{\text {th }}$ edition staging system, the LRFS curves of T1 and T2a showed a trend toward overlap, while better separation was observed between $\mathrm{T} 1$ and $\mathrm{T} 2$ in the $7^{\text {th }}$ edition. However, no significant differences of LRFS were observed between classifications T1 and T2 according to the $7^{\text {th }}$ edition. T2a patients had similar recurrent rates with $\mathrm{T} 1$ patients, according to the $6^{\text {th }}$ edition system. Therefore, a reclassified the previous T2a to T1 would be more reasonable.

The criteria for defining masticator space were disputed before the $7^{\text {th }}$ edition was published. Anatomically, the masticator space contains the medial and lateral pterygoid muscles, the masseter muscle and the temporalis muscle, as described in radiology textbooks ${ }^{[17-18]}$. However, masticator space in the $6^{\text {th }}$ edition was defined asextension beyond the anterior surface of the lateral pterygoid muscle or beyond the posterolateral wall of the maxillary antrum and/or the pterygo-maxillary fissure ${ }^{[19]}$. The study of Tang et al. showed anatomic masticator space involvement affected the overall survival and local relapse-free survival of NPC, and the authors recommended the anatomic masticator space involvement be categorized as classification $\mathrm{T} 4$. Similar conclusion was drawn from our study. Our data showed that anatomic masticator space involvement was identified as an independent prognostic factor for overall survival and progress free survival. As IMRT can provide more accurate dose distribution for targets and protection for important tissues, patients with infratemporal fossa involvement could get excellent radiotherapy efficacy. We proposed that anatomic masticator space should be taken into the staging system as a whole in the area of IMRT.

In our study, LRFS curves of classifications T3 and $\mathrm{T} 4$ in the $7^{\text {th }}$ edition staging system had a trend of mergence. It also revealed a lack of hazard discrimination for LRFS between classifications T1 and T2, T3 and T4, and a borderline significance for LRFS between T2 and T3(p=0.051). It is considered that the application of advanced diagnostic and therapeutic facilities and concurrent chemotherapy plus radiotherapy have contributed to this result. MRI can accurately indicate the primary tumor extension of NPC. Three-dimensional conformal radiotherapy and intensity-modulated radiotherapy have remarkable advantages in ensuring adequate dosage delivered to tumor and avoiding damages of tissues. The combination of 3D-CRT/IMRT with chemotherapy has increased the local control of NPC. As a result, $T$ stage would have less effect on prognosis [21-22], thus, it would be necessary to simplify the staging system in the future. We trailed to merge $\mathrm{T} 1$ and T2 into T1 (hence T3 and T4 would become T2 and T3), T3 and T4 into T3 respectively, and analyzed survivals of these proposed staging system. A better separation of LRFS curves and hazard discrimination were observed when stages T1 and T2 were merged into T1. It seemed that it was more reasonable to classify the current $\mathrm{T} 2$ to $\mathrm{T} 1$.

For $\mathbf{N}$ categories, retropharyngeal lymph node (RLN), as the first nodal station for regional spread of NPC, has a high metastatic frequency [23]. RLN invasion, regardless of unilateral or bilateral location, is categorized as N1 by MRI in the $7^{\text {th }}$ edition staging system. But the prognostic value of RLN metastasis in patients with NPC is controversial. Ma, Pan and Tham et al. [24-26] demonstrated that RLN metastasis affects the DMFS rates of patients with NPC. The study of Tang ${ }^{[27]}$ showed that no significant difference was observed between patients with and without RLN metastasis. In our study, the frequency of retropharyngeal lymph node metastasis was $56.1 \%$. Significant differences of DMFS between classifications N0 and N1, classifications N1 and N2 were obtained. However, multivariate analysis showed RLN metastasis only had influence on OS, but no effect on DMFS and PFS. We supposed the main reason was RLN could probably get relatively high dosage, due to its location which was adjacent to the primary nasopharyngeal tumor, especially using IMRT. The use of combined chemotherapy also reversed the effect of RLN metastasis on DMFS. The proportion of N3a and N3b in our data were $18(2 \%)$ and 30(3.3\%), respectively. These groups had worse prognosis than other $\mathrm{N}$ categories, but there was no significance between N3a and N3b for distant failure 
$(\mathrm{P}=0.59)$, which is similar to lee's study ${ }^{[11]}$. Data from Yue ${ }^{[28]}$ also showed that patients with classifications N3a and N3b had similar prognosis. We propose that it is not necessary to subdivide $\mathrm{N} 3 \mathrm{a}$ and $\mathrm{N} 3 \mathrm{~b}$ subgroups. Modification to merge N3a and N3b is reasonable to simplify the current staging system.

\section{Study limitations}

Our study comprised data from one center between 2004 and 2005, with a relative long follow-up. However, due to unavoidable economic limitations at that time, only $144(15.9 \%)$ patients in our cohort underwent 3D-CRT/IMRT, and it might have a negative influence on evaluating the staging system accuracy when IMRT is the main radiation modality for NPC worldwide. Plenty of prognostic factors, such as EBV-DNA levels, tumor volume, biological and genetic factors are being studied presently. These factors and more relevant additional prognostic factors should be identified and validated to refine the present TNM system. As a retrospective study, our conclusions need to be confirmed by prospective studies with a relatively large cohort.

\section{Conclusions}

In summary, the $7^{\text {th }}$ edition staging system is better at predicting prognosis. It has an even distribution and good separation of survival curves among different stages. $\mathrm{T}$ classifications and $\mathrm{N}$ classifications were independent prognostic factors for most of the endpoints.

\section{Acknowledgments}

We thank WeiHan HU (Department of Radiation Oncology, Sun Yat-sen University Cancer Centre) for contributing patients to the study, assistance with the data management, and logistic support. We thank the Collaborative Innovation Center of Cancer Medicine, Sun Yat-sen University Cancer Centre, for data management, and statistical assistance. We thank our patients and their families for their willingness in this study.

\section{Funding}

This study was funded by the Science and Technology Department of Guangdong Province (grant number: A2015112).

\section{Ethical approval}

All procedures performed in studies involving human participants were in accordance with the ethical standards of the institutional and/or national research committee and with the 1964 Helsinki declaration and its later amendments or comparable ethical standards.

\section{Competing Interests}

The authors have declared that no competing interest exists.

\section{References}

1. Wee JT, Ha TC, Long SL, et al. Is nasopharyngeal cancer really a "Cantonese cancer"? Chin J Cancer. 2010, 29(5):517-526.

2. Lam KO, Lee AW, Choi CW, et al. Global Pattern of Nasopharyngeal Cancer: Correlation of Outcome with Access to Radiation Therapy. Int I Radiat Oncol Biol Phys. 2016 ,94(5):1106-1112

3. Ma BB, Hui EP, Chan AT. Systemic approach to improving treatment outcome in nasopharyngeal carcinoma: current and future directions. Cancer Sci. 2008, 99(7):1311-1318.

4. Liu MT, Hsieh CY, Chang TH, et al. Prognostic factors affecting the outcome of nasopharyngeal carcinoma. Jpn J ClinOncol, 2003, 33(10):501-508.

5. Sun $\mathrm{Y}$, Tang LL, Chen $\mathrm{L}$, et al. Promising treatment outcomes of intensity-modulated radiation therapy for nasopharyngeal carcinoma patients with N0 disease according to the seventh edition of the AJCC staging system. BMC Cancer. 2012,15(12):2407-2412.

6. Cooper JS, Fleming ID, Henson DE. American Joint Committee on Cancer manual for staging of cancer; 6th ed. Philadelphia: JB Lippincott, 2002.

7. Lee AW, Sze WM, Au JS, et al. Treatment results for nasopharyngeal carcinoma in the modern era: The Hong Kong experience. Int J Radiat Oncol BiolPhys 2005, 61(4):1107-1116

8. Edge SB, Byrd DR, Compton CC. AJCC cancer staging handbook from the AJCC cancer staging manual, 7th edn. Springer, New York, 2011, 38(2):408-408

9. Sobin LH, Gospodarowicz MK, Wittekind C. TNM Classification of malignant tumors (UICC International Union Against Cancer) $7^{\text {th }}$ ed. Oxford: Wiley-Blackwell; 2009

10. Chen L, Mao YP, Xie FY, et al. The seventh edition of the UICC/AJCC staging system for nasopharyngeal carcinoma is prognostically useful for patients treated with intensity-modulated radiotherapy from an endemic area in China. Radiother Oncol. 2012, 104(3):331-337.

11. Lee AW, Ng WT, Chan LK, et al. The strength/weakness of the AJCC/UICC staging system (7th edition) for nasopharyngeal cancer and suggestions for future improvement. OralOncol. 2012, 48(10):1007-1013.

12. Tang L, Li L, Mao Y, et al. Retropharyngeal Lymph Node in Nasopharyngeal Carcinoma: Prognostic Value and Staging Categories. Clin Cancer Res 2007, 13 (5): $1445-1452$.

13. Luo W, Deng XW, Lu TX. Dosimetric evaluation for three dimensional conformal, conventional, and traditional radiotherapy plans for patients with early nasopharyngeal carcinoma. Ai Zheng 2004; 23 (5):605-608.

14. OuYang PY, Shi D, Sun R, et al. Effect of intensity-modulated radiotherapy versus two-dimensional conventional radiotherapy alone in nasopharyngeal carcinoma. Oncotarget. 2016;7(22):33408-17.

15. Liu MZ, Tang LL, Zong JF, et al. Evaluation of Sixth Edition of AJCC staging system for nasopharyngeal carcinoma and proposed improvement. Int J Radiat Oncol BiolPhys, 2008, 70(4):1115-1123

16. Mao YP, Xie FY, Liu LZ, et al. Re-evaluation of the 6th Edition of the AJCC staging system for nasopharyngeal carcinoma and proposed improvement based on magnetic resonance imaging. Int J Radiat Oncol BiolPhys, 2009, 73(5):1326-1334

17. Chong VF, Fan YF. Pictorial review: radiology of the masticator space. Clin Radiol 1996;51(7):457-465.

18. Curtin HD. Separation of the masticator space from the parapharyngeal space. Radiology 1987; 163(1):195-204.

19. Greene FL, Page DL, Fleming ID, et al. AJCC Cancer Staging Manual; 6th edn. Springer, New York, 2002.

20. Cheng SH, Yen KL, Jian JJ, et al. Examining prognostic factors and patterns of failure in nasopharyngeal carcinoma following concomitant radiotherapy and chemotherapy: impact on future clinical trials. Int I Radiat Oncol BiolPhys, 2001, 50(3):717-726

21. Lee AW, Lau WH, Tung SY, et al. Hong Kong Nasopharyngeal Cancer Study Group. Preliminary results of a randomized study on therapeutic gain by concurrent chemotherapy for regionally-advanced nasopharyngeal carcinoma: NPC-9901 Trial by the Hong Kong Nasopharyngeal Cancer Study Group. J Clin Oncol,2005, 23(28): 6675-6966.

22. Perri F, Bosso D, Buonerba C, et al. Locally advanced nasopharyngeal carcinoma: current and emerging treatment strategies. World J Clin Oncol, 2011, 2(12):377-383.

23. Ho FC, Tham IW, Earnest A, et al. Patterns of regional lymph node of metastasis of nasopharyngeal carcinoma: a meta-analysis of clinical evidence. BMC cancer, 2012, 12(1):98

24. Ma J, Liu L, Tang L, et al. Retropharyngeal lymph node metastasis in nasopharyngeal carcinoma: prognostic value and staging categories. Clin Cancer Res, 2007, 13(5): 1445-1452. 
25. Pan J, Xu Y, Qiu S, et al. A comparison between the Chinese 2008 and the 7th edition AJCC staging systems for nasopharyngeal carcinoma. Am J Clin Oncol, 2015, 38 (2):189-196

26. Tham IW, Hee SW, Yap SP, et al. Retropharyngeal nodal metastasis related to higher rate of distant metastasis in patients with $\mathrm{N} 0$ and $\mathrm{N} 1$ nasopharyngeal cancer. Head Neck, 2009, 31(4):468-474.

27. Guo Q, Pan J, Zong J, et al. Suggestions for lymph node classification of UICC/ AJCC staging system: a retrospective study based on 1197 nasopharyngeal carcinoma patients treated with intensity- modulated radiation therapy. Medicine (Baltimore).2015 May; 94(20):e808.

28. Yue $\mathrm{D}, \mathrm{Xu} \mathrm{YF}, \mathrm{Zhang} \mathrm{F}$, et al. Is replacement of the supraclavicular fossa with the lower level classification based on magnetic resonance imaging beneficial in nasopharyngeal carcinoma? Radiother Oncol. 2014,113(1):108-14. 